\title{
SLA for E-Learning System Based on Cloud Computing
}

\author{
Doaa Elmatary \\ Industrial Technical Institute \\ Technical college, Port Said \\ Port said, Egypt
}

Samy Abd El Hafeez

Department of Mathematics and Computer Science

Faculty of Science, Port Said University

Port Said, Egypt

\begin{abstract}
The Service Level Agreement (SLA) becomes an important issue especially over the Cloud Computing and online services that based on the 'pay-as-you-use' fashion. Establishing the Service level agreements (SLAs), which can be defined as a negotiation between the service provider and the user, is needed for many types of current applications as the E-Learning systems. The work in this paper presents an idea of optimizing the SLA parameters to serve any E-Learning system over the Cloud Computing platform, with defining the negotiation process, the suitable frame work, and the sequence diagram to accommodate the E-Learning systems.
\end{abstract}

Keywords-Cloud Computing; Service Level Agreement (SLA); E-learning System

\section{INTRODUCTION}

The Cloud Computing defined as delivering the hosted services over the Internet in an on-demand model. A cloud provider offers resources (e.g., hardware, software or development stacks) as services over the internet depending on a pay-as-you-use basis [1]. In the Cloud Computing, the users could access applications and services by using his browser no matter where these applications and services are hosted [1]. Using the applications of the Cloud Computing provides the user an effective way to use the infrastructure, and to maintain his services where he does not need to waste his time in installing and maintaining any components on his device [2].

The Cloud Computing Models can be divided into three layers, every layer handle a certain service; Software as a Service (SaaS), Platform as a Service (PaaS), and Infrastructure as a Service (IaaS) (see Figure [1]) [3]. Software as a service ( $\mathrm{SaaS}$ ) eliminates the need for installing and running the application on the local devices of users, so that the users can access the network and the available programs remotely via internet [2]. Platform as a service (PaaS) provides the necessary framework to develop the applications without the cost and complexity of buying and managing the underlying hardware [3]. Infrastructure as a service (IaaS) offers the required physical infrastructure (e.g., servers, storage, processors) [2].

\author{
Wael Awad \\ Department of Mathematics and Computer Science \\ Faculty of Science, Port Said University \\ Port Said, Egypt
}

Fatma Omara

Department of Computer Science, Faculty of Computers and Information, Cairo University, Cairo, Egypt

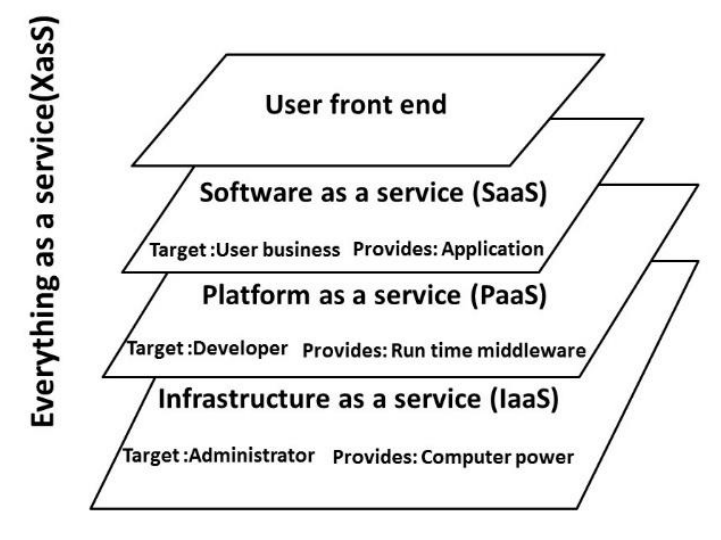

Fig. 1. Architecture of Cloud Computing Layers [4]

One of the main aspects in the Cloud Computing is isolating the user from the internal details of the system, which means that there is no control from the user on the computing resources. Thus, the user needs to guarantee the resources availability and reliability, this guarantee is provided through a negotiation between the provider and consumer to create a contract with some criteria called Service Level Agreements (SLAs) [2].

On the other hand, the SLA is a contract between the consumer and the provider. The main issue of SLAs is to provide a clear definition of the formal agreements about the service terms like performance, availability and billing. It is important that the SLA includes the obligations and actions that can be taken in the case of any violation, with clearly defined semantics between each party involved in the online contract [6].

As the SLA becomes an important issue for any user over the cloud, it can be used for different kinds of applications (e.g., medical, commercial and learning systems). According to the work in this paper, the SLA is applied on the ELearning system that serves a different kind of users. 


\section{RELATED WORKS}

Selecting a Template (Heading 2) The SLA has been used since the late 1980 s by telecom operators as a part of the contract with their customers. After that, the SLA becomes a standard protocol of business applications and Web Services [9]. Generally, There are two main specifications are designed to describe the SLA; 1) The Service Negotiation and Acquisition Protocol (SNAP) which support reliable management of remote SLAs and describe the negotiating process in the system [10]. 2) The conceptual SLA frame work for Cloud Computing that describes the main characteristics of SLAs in Cloud Computing and explains the SLA parameters specified by metrics for the four types of cloud services (i.e., IaaS, SaaS, PaaS, Storage as a Service) [6]. Chenkang,W.,et. al [11] have suggested different implementation structure of SLAs parameters by adding another dimension related to both of the providers and the users. Concerning the IaaS, the SLAs represent the metrics of the performance for the provider, and the experience for the users. While in the PaaS, both providers and users need more guarantees for the integration's ability and scalability. Finally, for SaaS, the providers need to offer some specialized functions like multi-terminal supporting and customization, and the users should care about the stable usability.

Patel, P., et. al. [12] has proposed a framework with a main concept and architecture for the Web Service Level Agree (WSLA). It is a contract defines the web services where the cloud provider is out of their scope.

All of the previous researches handled the SLA in general without applying its principles on a specific application by describing how the cloud services are offered to the customers using SLA (Service Level Agreement).

On the other hand, Shrivastava, G. [13] has applied the Cloud Computing to serve the E- Learning system. The availability of online resources in the Cloud Computing gives the student the opportunity to share the E-Learning resources and access it online at anytime and anywhere.

Finally, this research is based on the idea of optimizing the SLA parameters, negotiation process, and the frame work to accommodate the E-Learning systems. Building the ELearning server on Cloud Computing gives the ability to adjust the required resources as needed, and allows the users to get the benefits of Cloud Computing services.

\section{THE SERVICE LEVEL AgREEMENTS (SLAS)}

The SLA is a legal format to document the way of delivering the service, as well as, providing a framework for paying service charges. Both the providers and users dealwith SLAs from their points of view. From the Service provider's side, he uses the contract to optimize his use of infrastructure to meet the signed terms of the services. While, the Service user deals with the SLA to ensure the required level of service quality and to maintain acceptable business models for long-term provisioning of services [6]. The main requirements of the SLAs can be concluded as follows [6]:
- SLA's format should clearly describe a service so that the service's user can easily understand the operations that can be done on the services.

- SLA has to present the level of performance of service.

- SLA has to define the ways of monitoring the service parameters and the format of reports monitoring.

- SLA has to clarify the penalties when the service requirements are not met.

- SLA presents the business metrics such as billing values when this service is done without any penalties.

The SLA parameters are specified by metrics; these metrics define how the parameters of the cloud service can be measured. Usually, these metrics are varied from an application to another. So in this paper, the SLA parameters are specified only for the E-Learning applications [6]. Most users are confused in defining the important parameters. For the E-Learning applications, four types of services which providers can provide to the users. These services are IaaS, SaaS, PaaS, and Storage as a Service [6]. For each part of the SLA, the most important parameters that the users can use to create a reliable model of negotiation with the service provider are defined [6].

This paper focuses on the most influence parameters that will serve any E-Learning system. Where the E-Learning system will be created based on the Cloud Computing, thus all the required resources can be adjusted as needed.

Table (1) represents the SLA metrics of SaaS for the ELearning system

TABLE I. SLA METRICS FOR SAAS

\begin{tabular}{|l|l|}
\hline Parameter & Description for the Educational Service \\
\hline Reliability & The system can be still working in most cases. \\
\hline Usability & Easy and Clear Interface \\
\hline Scalability & Using with one user only or with all users. \\
\hline Customizability & Flexible to use with different type of users. \\
\hline
\end{tabular}

Table (2) represents one of the most important parameters in the SLA metrics of IaaS for the E-Learning system, where a sufficient area (storage) has to be created to keep the required data of the system whether for short or long contract agreement. This can be done by reserving a storage unit from the Cloud provider.

TABLE II. SLA METRICS FOR IAAS

\begin{tabular}{|l|l|}
\hline Parameter & \multicolumn{1}{|c|}{ Description for the Educational Service } \\
\hline Storage & $\begin{array}{l}\text { Storage "Z" (big) size of data for the organization. } \\
\text { Storage "Z*" (small) size of data for the student. }\end{array}$ \\
\hline
\end{tabular}

Table (3) represents the SLA metrics of Storage as a Service that should be a guarantee for the E-Learning system.

PaaS can be provided to extend the E-Learning system by offering extra services to all users. So, the software applications have to access the platform by using the user defined browsers [8]. The SLA metrics of PaaS of E-Learning system are displayed in Table (4). 
TABLE III. SLA METRICS FOR STORAGE AS A SERVICE

\begin{tabular}{|l|l|}
\hline Parameter & \multicolumn{1}{|c|}{ Description for the Educational Service } \\
\hline $\begin{array}{l}\text { Storage } \\
\text { billing }\end{array}$ & $\begin{array}{l}\text { For the price billing, the most influence units are the } \\
\text { processing and storage. }\end{array}$ \\
\hline Security & $\begin{array}{l}\text { No one can access the material without registration and } \\
\text { authentication. That is to prevent the system from the } \\
\text { illegal access. }\end{array}$ \\
\hline Privacy & (validation authentication,verification) \\
\hline Backup & $\begin{array}{l}\text { Images of data (database of the users' profiles) are stored } \\
\text { according to the regulation of the E-learning system, and } \\
\text { users have the ability of recovery the profile data in case of } \\
\text { disaster occurrence. }\end{array}$ \\
\hline
\end{tabular}

TABLE IV. SLA METRICS OF PAAS FOR THE E-LEARNING SYSTEM

\begin{tabular}{|l|l|}
\hline Parameter & \multicolumn{1}{c|}{ Description for the Educational Service } \\
\hline Integration & Coordination with other institutions to extend the platform. \\
\hline Scalability & Extends itself so estimated offering services to all users. \\
\hline $\begin{array}{l}\text { Pay as you } \\
\text { go billing }\end{array}$ & Pay only for the service that you use. \\
\hline Browsers & User defined \\
\hline
\end{tabular}

\section{THE PROPOSED SLA FOR E-LEARNING SYSTEM}

The proposed SLA for E-Learning system represents the metrics should be defined for the "E-Learning system" over the Cloud Computing. At this moment, the SLA negotiation process between the students and the instructors is needed to be explained. The frame work of the E-Learning's SLA is shown in Figure (2). It consists of three entities; Parties, Service definition, and Obligation Services.

\section{A. Parties}

It consists of the parties of the agreement. The service's provider deals with the coordinator of the E-Learning system who is considered here the service user. Then, both the student and the instructor deal with the coordinator, where he is considered the service provider. So, the coordinator could be considered as the service broker.

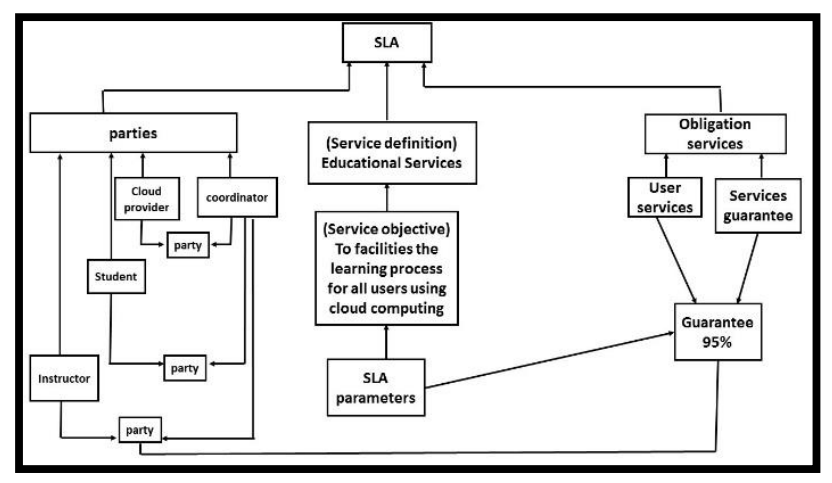

Fig. 2. Frame Work of SLA for E-Learning System

\section{B. Service Definition}

It defines the educational service and its objective to improve the facilities of the learning process for the users (i.e. students or instructors) by using the Cloud Computing environment. This component contains SLA parameters for the service. As shown in Figure (3), it has two main cloud metrics (i.e. infrastructure as a service and Storage as a service).

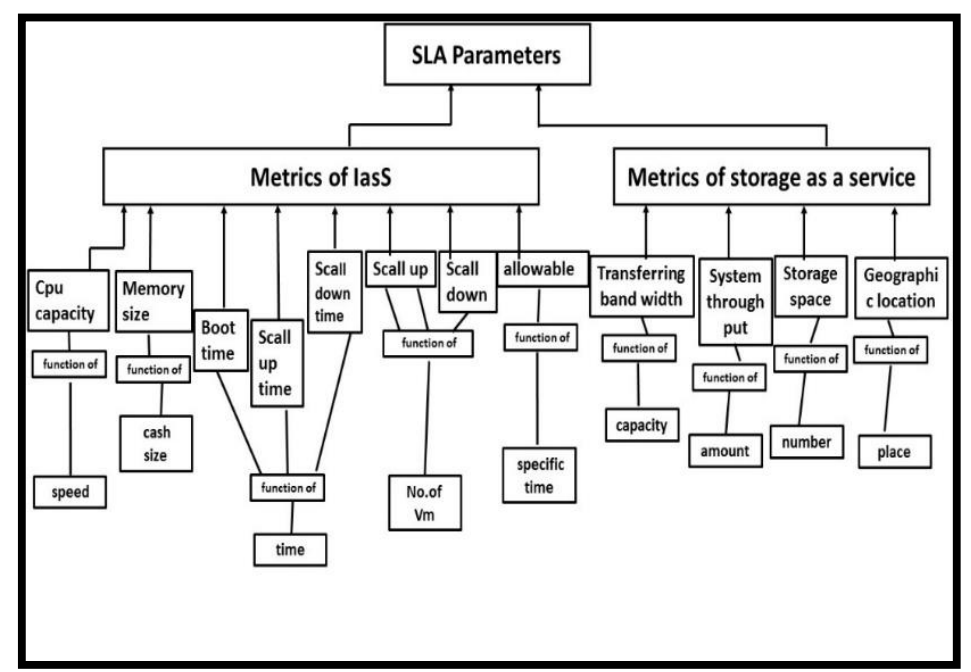

Fig. 3. SLA of Service Definition

\section{Obligation Services}

It contains the service guarantee represented in common parameters for all of the users (students and instructors) to ensure the services that are delivered (see Figure (4)).

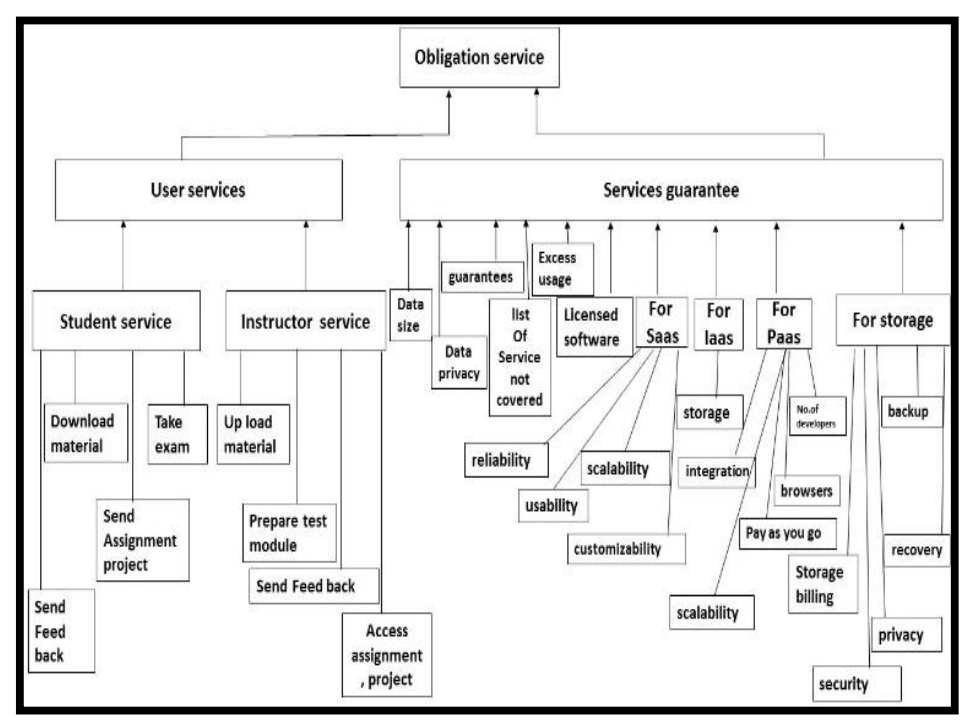

Fig. 4. Obligation Services of SLA for E-Learning System

\section{SLA RELATIONAL DIAGRAM FOR THE E-LEARNING SYSTEM}

Relational diagram (RD) is a graphical representation of an information system that shows the relationship between people, objects, places, concepts or events within that system [14].

The three main components of the RD are; 1) the entities, which are objects or concepts that store the information, 2) the relationship between those entities, and 3) the cardinality, which defines that relationships in terms of numbers [14]. The proposed Relational Diagram consists of four components; cloud provider, negotiation process, system implementation, and SLA document (see Figure (5)). 


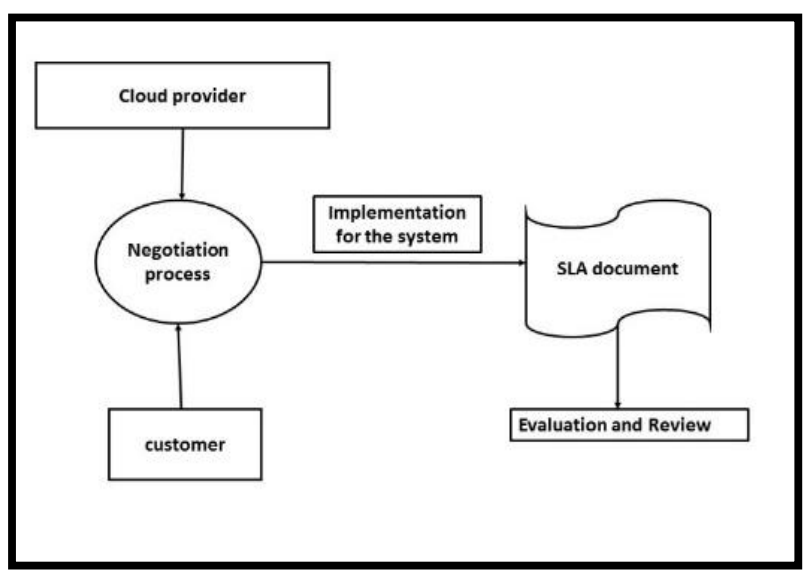

Fig. 5. SLA Relational Diagram for E-Learning System

\section{A. Negotiation Process}

Between the service's provider and customer, there is a negotiation process using a Service Negotiation and Acquisition Protocol (SNAP) and depending on different scenarios. In the first scenario, all the users ask for the agreement using the agreement state transitions (where the coordinator plays the role of the service's broker). The two other scenarios are:

- Community Scheduler, which is an entity that acts as an intermediary between the community and its resources, and

- File Transfer; it restricts the activity of sending and receiving requests from/to the user (e.g. transferring a file) with a deadline time [10].

These two Scenarios are used to negotiate with the ELearning services.

Figure (6) shows the negotiation process using the community scheduler for the process of uploading the material book, which is done by the instructor for the student where:

- Task Service Level Agreements (TSLAs) is the procedure that concerns with the task of the uploading process. So, TSLA is "Uploading Process".

- Resource Service Level Agreements (RSLAs) where it defines the available resources in the system. It depends on the procedure of the TSLA. So, the RSLAs is "RSLA Resources".

- Binding Service Level Agreements (BSLAs); it is the binding agreement to apply the procedure of a service. So, the BSLA is: "upload the resources".

The activities diagram for the proposed SLA for the ELearning system consists of three entities; the instructor ' $i$,' the student ' $u$ ', and the scheduler ' $\mathrm{s}$ ', where both the student and the instructor perform the task by sending their activity, and the scheduler manages the system during the process.

Figure (6) shows the material activities that consist of two main processes, uploading the material by the instructor, and download it by the student.
According to Figure (6), each user sends activation to the community scheduler to perform the task whether from the instructor $(i)$ or student $(u)$, where:

TSLA: upload/download process.

RSLA: content Materials.

BSLA: upload/download resources.

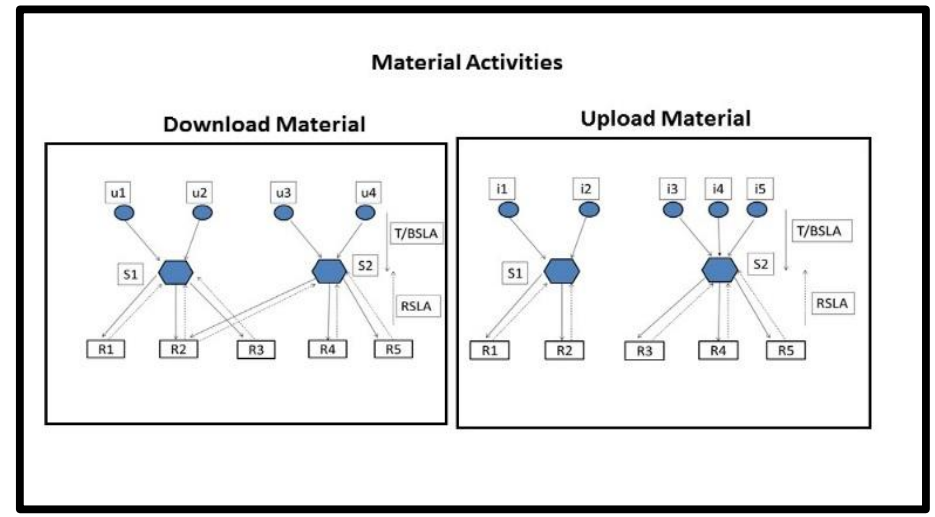

Fig. 6. Material Activities for E-Learning System

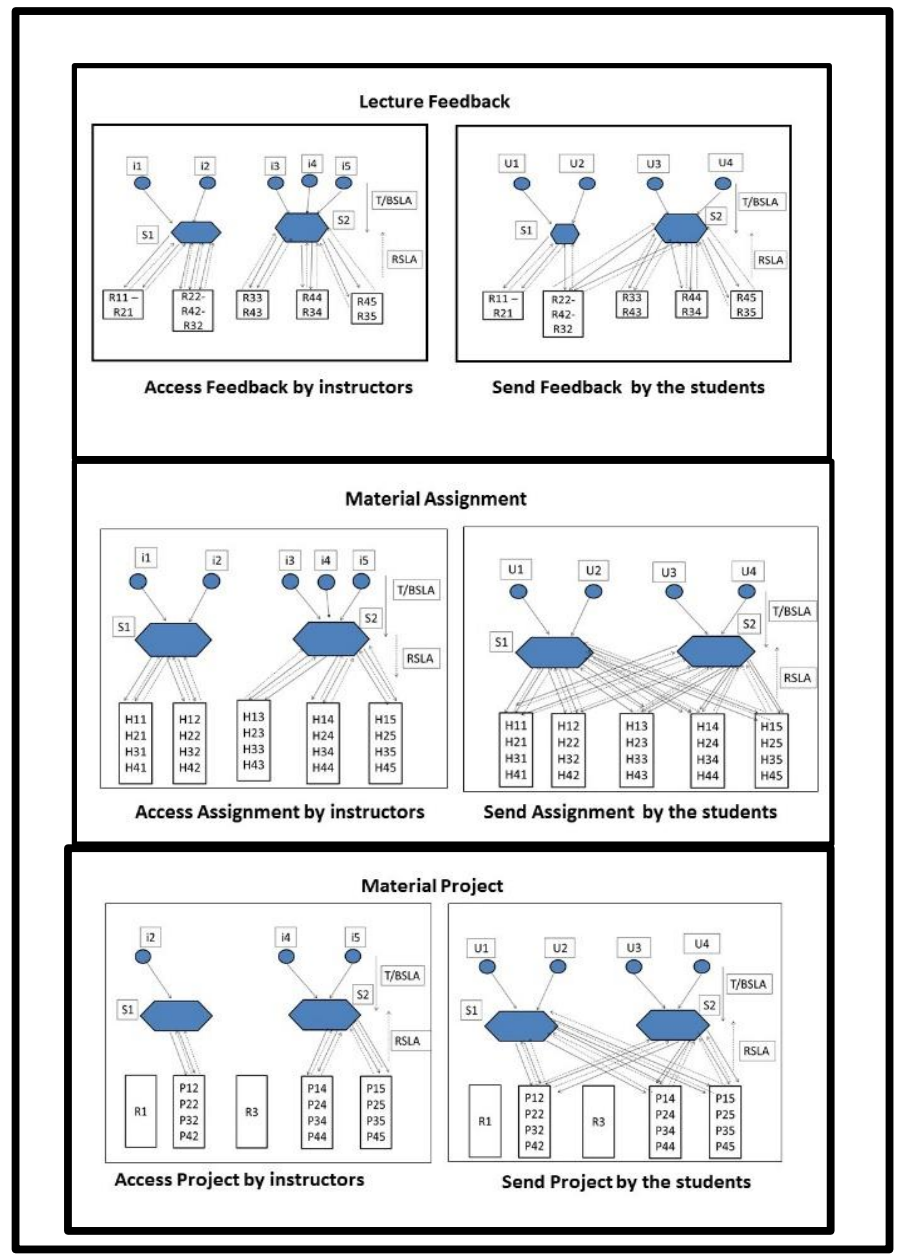

Fig. 7. Academic Activities For E-Learning System 


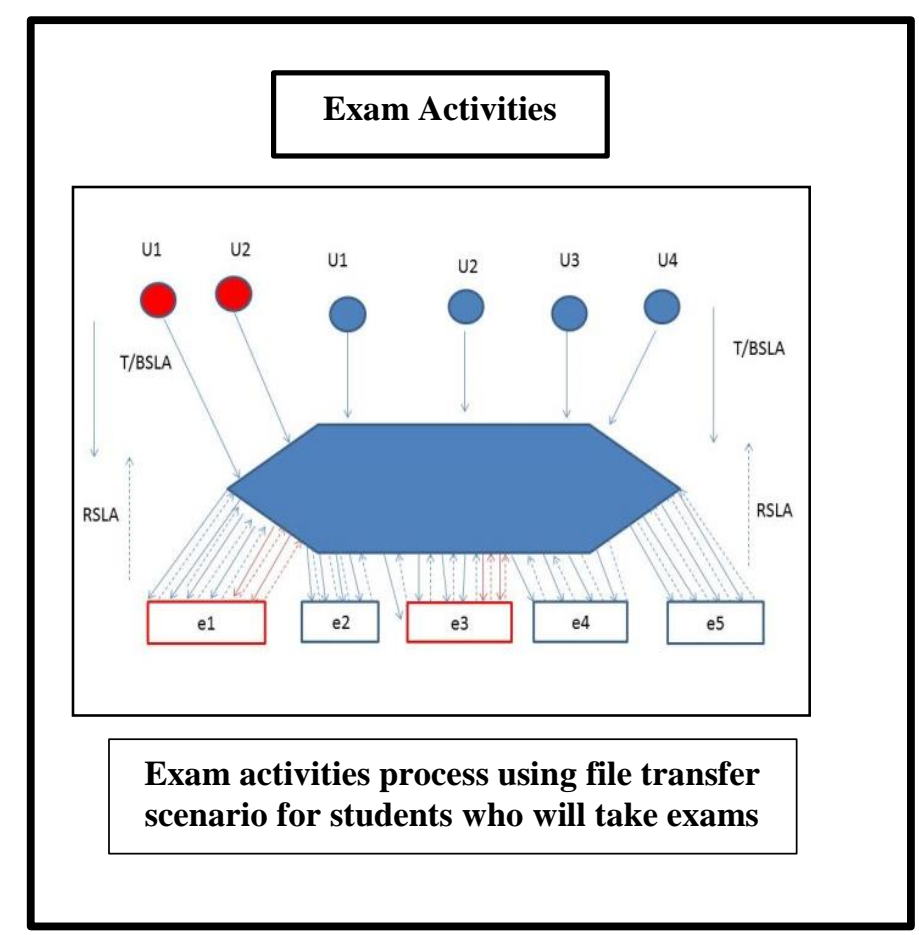

Fig. 8. Exam Activities for E-Learning System
Figure (7) represents the academic activities diagram for the proposed E-Learning system. It consists of three activities that are the lecture feedback, the material assignment, and the material project. For these activities, the student $(U)$ sends an activation for the community scheduler $(S)$ to perform the TSLA (i.e. the sending process), and also the instructor $(i)$ sends an activation to access the RSLA (i.e. resources). Finally, the BSLA is performed by sending the lecture feedback, Material assignment, and Material project.

Figure (8) represents the exam's activity in the E-Learning system as an example. The user submits the job to the scheduler within a deadline time, and then the scheduler reserves a storage space on the destination resource to ensure there is enough space for the current activity before beginning the transfer. The file transfer scenario handle achieving this activity. Once the space is allocated, the scheduler reserves the suitable bandwidth from the network.

\section{Sla FrameWork SEQUENCE Diagram}

The sequence diagram is an interaction diagram shows how the service's processes operate with one another with defining their orders [15]. Figure (9) describes the SLA frame work sequence diagram that explains the sequence of the services for all users (i.e. students, instructor, and the coordinator).

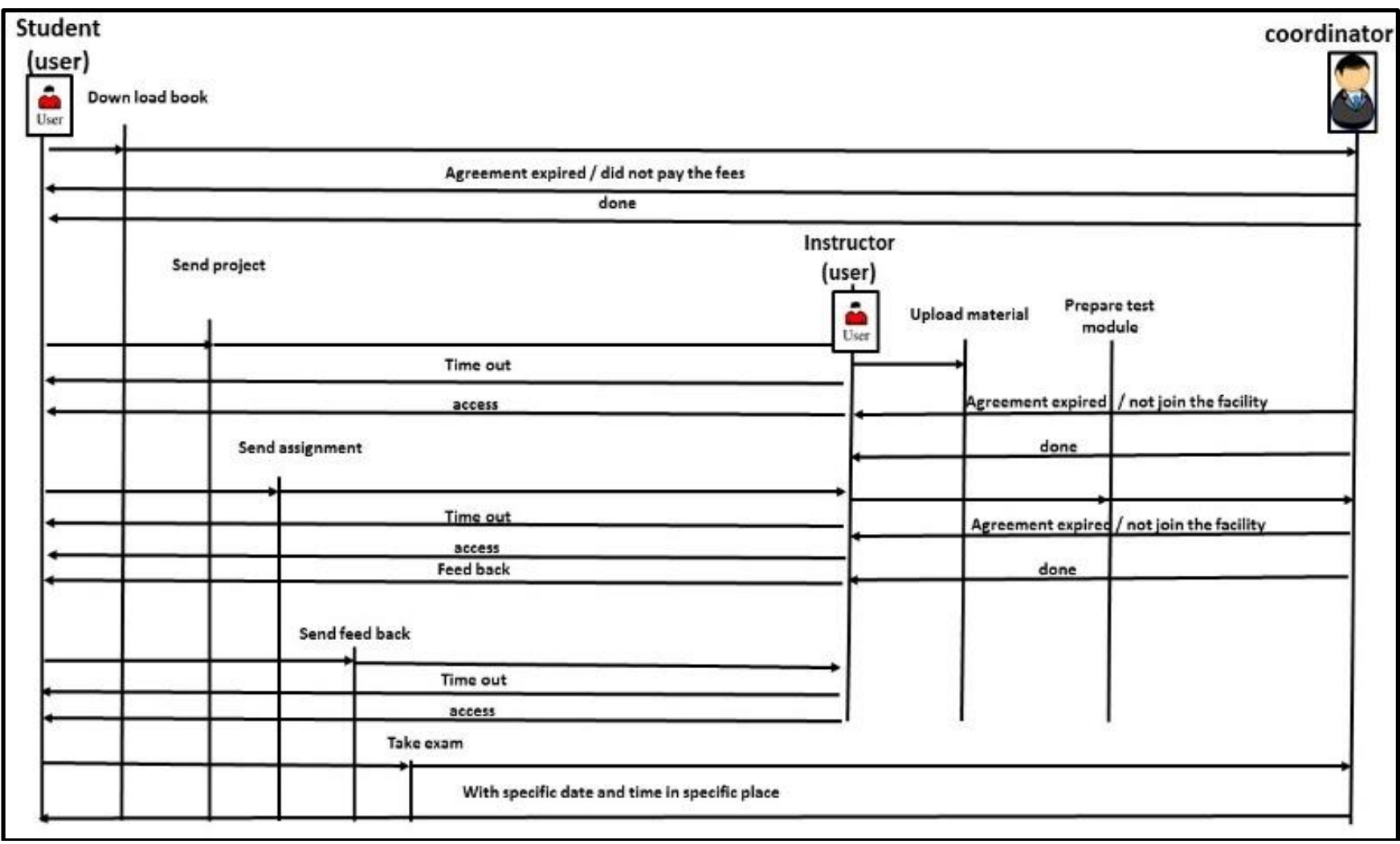

Fig. 9. Frame Work Sequence Diagram

\section{CONCLUSION AND FUTURE WORK}

An effective Service Level Agreement (SLA) is the way to guarantee the process of the service providing from the cloud provider to the cloud user. The more clear definition of the
SLA parameters gives more flexibility, confidence, and reliability between the provider and the user. The main goal of this paper is to represent the service level agreement (SLA) for an E- learning system through defining the influential metrics of SLA for SaaS, IaaS, PaaS, and Storage as a 
Service, and designing the framework, sequence diagram and architecture of the proposed system.

The E-Learning system implementation and SLA document (terms and conditions) will be considered as a future work.

\section{REFERENCES}

[1] Dillon, T., and W, C.," Cloud Computing: Issues and Challenges", IEEE International Conference on Advanced Information Networking and Applications, Vol.24, PP. 27-29, 2010.

[2] Jadeja, Y, and Modi, K" Cloud Computing-Concepts,Architecture and Challenges " International Conference on Computing, Electronics and Electrical Technologies (ICCEET), PP. 877-899, 2012.

[3] Prasanth, A.," Cloud Computing Services: A Survey", International Journal of Computer Applications, Vol. 46, No. 3, May 2012

[4] Pallis, G. "Cloud Computing the New Frontier of Internet Computing", IEEE Computer Society,PP.71-75,2010.

[5] Zhang,Q. and Boutaba ,R.," Cloud Computing: state-of-the-art and research challenges", Journal of Internet Services and Application, Vol.1,Issue1, PP. 7-18, 2010

[6] Alhamad, M., Dillon,T., and Chang,E.," Conceptual SLA Framework for Cloud Computing", IEEE International Conference on Digital Ecosystems and Technologies, vol. $4^{\text {th }}$, PP. 606-609, TBD Dubai, United Arab Emirates 2010.

[7] POCATILU, P., Alecu, F.,and Vetrici, M.," Using Cloud Computing for E-learning Systems", Published in8th WSEAS international conference on Data networks, communications, computers,PP.54-59from November 7-9-2009
[8] Mathew,S., "implementation of Cloud Computing in education - A evolution", International Journal of Computer Theory and Engineering, Vol. 4, No. 3, pp. 473, June 2012.

[9] Armbrust, M., Fox,A., Griffith,R., Joseph,A., Katz,R., Konwinski,A., Lee,G., Patterson,D., Rabkin,A., Stoica,I.,and Zaharia,M., "Above the Clouds: A Berkeley View of Cloud Computing",http://www.moonther.com/cis492/abovetheclouds.pdf

[10] Czajkowski,k.,Foster,I., Kesselman,C., Sander,V.,and Tuecke,S., SNAP: A Protocol for Negotiating Service Level Agreements and Coordinating Resource Management in Distributed Systems", Job Scheduling Strategies for Parallel Processing, Lecture Notes in Computer Science, Vol 2537, pp 153-183, 27 November 2002.

[11] Chenkang, W., Zhu,Y., and Pan,S., "The SLA Evaluation Model for Cloud Computing", International Conference on Computer, Networks and Communication Engineering, PP. 331-332, Beijing, China May 2324, 2013.

[12] Patel,P ., A. Ranabahu, and A. Sheth , "Service Level Agreement in Cloud Computing" kno.e.sis puplications http://knoesis.wright.edu/library/download/OOPS LA_cloud_wsla_v3.pdf (2009)

[13] Shrivastava, G.," E- Learning Improved Architecture for Clouds," Global Journal of Computer Science and Technology Cloud and Distributed, Vol. 13 Issue 2, PP. 33-35, 2013

[14] Rouse.M,http://searchcrm.techtarget.com/definition/entity-relationshipdiagram, Posted in October 2014, last access on 22.8.2015 at 1:39am.

[15] Moreia, A., Araújo,A.,and Brito,I.," Crosscutting Quality Attributes for Requirements Engineering", Software Engineering and Kno wledge Engineering Conference(SEKE), ISBN:1-58113-556-4,PP 167-174, Ischia, Italy, 15-19 July 2002. 